\title{
CONCERNING APOSYNDETIC AND NON-APOSYNDETIC CONTINUA
}

\section{F. BURTON JONES}

Introduction. One might judge from the title that $\mathrm{I}$ am going to discuss continua. For is not a continuum either aposyndetic or nonaposyndetic? What $I$ intend to do is to consider continua from a certain point of view, and from this point of view continua may be classified in a rough sort of way. This system of classification (and the basic concept upon which it rests) is only in its infancy. Here then is a report upon the beginning rather than the completion of an interesting and, I trust, useful field of study.

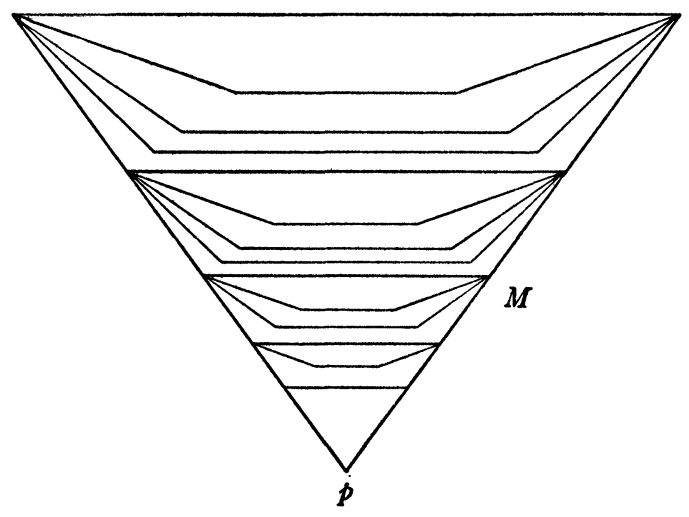

EXAMPLE 1

To avoid any confusion, I shall confine this discussion to continua lying in a compact metric space which in most cases is the complex number sphere (or a topological 2-sphere, $S^{2}$ ). Hence all continua are connected, closed, and compact sets.

Consider the difference between the familiar concepts of a continuum being connected im kleinen at a point and a continuum being locally connected at a point. ${ }^{1}$ A continuum $M$ is locally connected at a point $p$ of $M$ provided that if $R$ is a region containing $p$, there exists a connected open subset $U$ of $M$ such that $R \supset U \supset p$. The continuum $M$ is connected im kleinen at $p$ provided that if $R$ is a region

An address delivered before the Summer Meeting of the Society at Boulder, Colorado, September 1, 1949, by invitation of the Committee to Select Hour Speakers for Summer and Annual Meetings; received by the editors December 8, 1951.

1 For the definition of certain terms and phrases see [11]. Numbers in brackets refer to the bibliography at the end of this paper. 
containing $p$, there exist a subcontinuum $H$ of $M$ and an open subset $U$ of $M$ such that $R \supset H \supset U \supset p$. Now as a point-wise property connectedness im kleinen is the weaker of the two. This may be seen in Example 1. In this example the plane continuum $M$ consists of a simple infinity of contiguous and similar trapezoids (which form a sequence converging to $p$ ) together with a simple infinity of arcs lying in the interior of each trapezoid as indicated. A little study will show that $M$ is connected im kleinen at the point $p$ but $M$ is not locally connected at $p .^{2}$

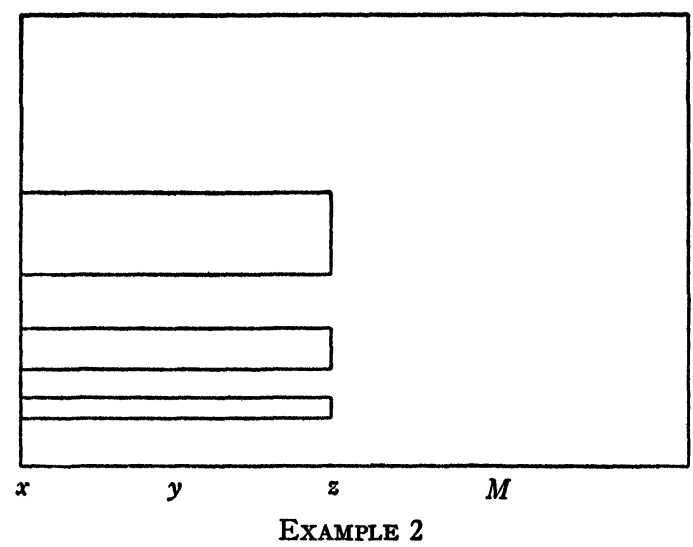

In order to generalize the weaker of these two notions, I shall rephrase the definition of connectedness im kleinen. Let $M$ be a continuum and let $G$ denote the collection of all closed subsets of $M$. Then $M$ is connected im kleinen at a point $p$ of $M$ provided that if $K$ belongs to $G$ and does not contain $p$, there exist a subcontinuum $H$ of $M$ and an open subset $U$ of $M$ such that $M-K \supset H \supset U \supset p$. Now let $G^{\prime}$ be the subcollection of degenerate elements of $G$, and substitute $G^{\prime}$ in the previous statement for $G$. Thus $M$ is aposyndetic at $p$ provided that if $k$ is a point of $M$ distinct from $p$, there exist a subcontinuum $H$ of $M$ and an open subset $U$ of $M$ such that $M-k$ $\supset H \supset U \supset p$. The term aposyndetic means bound-together-awayfrom. ${ }^{3}$ So for each point $k$ in $M-p, M$ is bound together at $p$ away from $k$ because the continuum $H$ lies in $M-k$ and contains a (relative) neighborhood of $p$.

It may be helpful to consider the following simple example in the plane (Example 2). In this example the continuum $M$ consists of a simple closed curve together with a simple infinity of rectangles as

2 An example of this nature has been given by Ayres in [1].

${ }^{8} \mathrm{Gk}$. apo $=$ away from, $\mathrm{syn}=$ together, deo $=$ to bind. See [4]. 
indicated, these rectangles forming a sequence which converges to the interval $x y z$. This continuum $M$ is aposyndetic at $x$, and $M$ is aposyndetic at $y$ with respect to $z$ but $M$ is not aposyndetic at $y$ with respect to $x$ because if $U$ is an open subset of $M$ containing $y$, any subcontinuum of $M$ which contains $U$ would have to contain $x$.

Boundary point theorems. The usefulness of the property of a continuum being aposyndetic at a point is illustrated by the following two theorems.

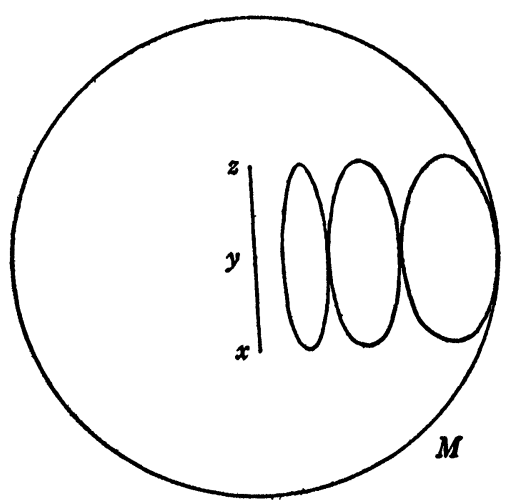

EXAMPLE 3

Theorem 1. In $S^{2}$ let $D$ be a complementary domain of a continuum $M$. Then $S^{2}-D$ is connected im kleinen at every point where $M$ is aposyndetic. ${ }^{4}$

A simple special case of Theorem 1 may serve to make the theorem clearer: If the continuum $M$ does not separate $S^{2}$, then $M$ is connected im kleinen at exactly those points where $M$ is aposyndetic. It does not follow that $M$ is locally connected at these points. In Example 1, throw away every point to the left of a vertical line through $p$. The remaining points form a continuum (not separating $\left.S^{2}\right)$ which is aposyndetic at $p$ but which is not locally connected at $p$.

However consider the following theorem:

TheOREM 2 (BING). If a continuum $M$ is not separated by any of its subcontinua, then $M$ is locally connected at every point where $M$ is aposyndetic [3].

Here is a test for local connectedness-at least in some cases.

4 Theorem 1 is a corollary of Theorem 10 in [4]. A complementary domain of a continuum is a component of its complement. The term domain will be used in general as equivalent to open set and is not necessarily connected. 
Return once more to a consideration of Theorem 1. Suppose that (in $S^{2}$ ) $D$ is a connected domain and $M$ is the boundary of $D$. Is $M$ connected im kleinen at every point where $M$ is aposyndetic? $N o$. For consider Example 3 in which $M$ is the sum of a simple infinity of tangent ellipses as indicated together with their sequential limiting set $x y z$. Even though $M$ is aposyndetic at $y, M$ is not connected im kleinen at $y$. But $M$ is not aposyndetic at $y$ with respect to $x+z$. This observation may be formalized as follows:

Theorem 3. Suppose that $y$ is a point of the boundary $M$ of a connected domain in $S^{2}$. Suppose further that if $x$ and $z$ are points of $M-y$ there exist a subcontinuum $H$ of $M$ and an open subset $U$ of $M$ such that $M-(x+z) \supset H \supset U \supset y$. Then $M$ is connected im kleinen at $y$.

Continuous curves, aposyndetic continua. If a continuum were aposyndetic at each of its points, one would guess that it would in many respects resemble a continuous curve (this is, a continuum which is connected im kleinen at each of its points and, hence, locally connected). This is particularly true of topological properties in the large. For instance consider the Torhorst Theorem.

TORHORsT THEOREM. The boundary of a complementary domain of a continuous curve in $S^{2}$ is itself a continuous curve [13].

The "continuous curve" in the hypothesis may be replaced by "aposyndetic continuum," so that we have the following:

Boundary TheOREM (WILDER-WhyBuRN). The boundary of $a$ complementary domain of an aposyndetic continuum in $S^{2}$ is a continuous curve $[4 ; 14 ; 17]$.

This, of course, follows immediately from Boundary Point Theorem 1. Priority of discovery belongs to Wilder for the cyclic case [16]. G. T. Whyburn proved the theorem independently for the general non-cyclic case [14]. Wilder's argument with slight modifications was extended by Wilder to the general case-and to higher dimensions [17]. My work generalized the theorem to more abstract twodimensional spaces and did away with some of the compactness requirements [4]. Wilder's and Whyburn's arguments are quite analogous and depend upon a sort of mass behavior of the points. So it may be instructive to compare the various notions involved here as well as the terminology.

Whyburn defined a continuum $M$ to be semi-locally-connected $(s-l-c)$ at a point $p$ provided that if $U$ is an open subset of $M$ containing $p$, there is an open subset $V$ of $M$ lying in $U$ and containing 
$p$ such that $M-U$ is covered by a finite number of components of $M-V$. Wilder used essentially the same idea. Wilder used the term almost $i$-avoidable ( $i$ being associated with dimension). As a pointwise property these notions are not directly related to connectedness im kleinen. For in Example 2, $M$ is s-1-c at $z$ where $M$ is not connected im kleinen and $M$ is connected im kleinen at $x$ where $M$ is not s-1-c. The situation will be a little clearer if the definition of s-1-c is rephrased as follows: the continuum $M$ is s-1-c at a point $x$ of $M$ if $M$ is aposyndetic at each point $y$ of $M-x$ with respect to $x$. So we have the following:

Equivalence Theorem. In order that a continuum $M$ be s-l-c it is necessary and sufficient that $M$ be aposyndetic [4].

As a point-wise property, the notion of semi-local-connectedness, while not yielding the boundary point theorem, is useful in another direction.

ACCessibility Theorem. If $D$ is a complementary domain of a continuum $M$ in $S^{2}$, then the boundary $B$ of $D$ is accessible from all sides from $D$ at those points of $B$ where $M$ is s-l-c.s

So the notions of a continuum being aposyndetic at a point and a continuum being s-l-c at a point are sort of complementary notions distilled from the notion of a continuous curve.

Now to return to the comparison of aposyndetic continua with continuous curves. Whyburn's main interest in aposyndetic continua was in connection with his cyclic element theory. In general, he has shown that for this theory aposyndetic continua (i.e., s-1-c continua) are just as satisfactory as continuous curves. In theorem after theorem in his book he has made this substitution [15].

A continuous curve in $S^{2}$ may be characterized by means of its complement [12]. There is an analogous theorem for aposyndetic continua.

Complement Theorem. In order that a continuum $M$ in $S^{2}$ be aposyndetic it is necessary and sufficient that its complement be nonfolded [5].

To give you an intuitive idea of what it takes to make a domain folded in the eyes of a topologist consider Example 2 again. There exists in $D$ an infinite sequence of crossed arc-segments (open arcs) $A_{i}+B_{i}$ whose endpoints lie on the boundary of $D$ such that the end-

\footnotetext{
${ }^{5}$ Neither $B$ nor $M$ is necessarily $s-l-c$ at every point where $B$ is accessible from all sides.
} 
points of the $B_{i}$ converge to a point not in the continuum to which

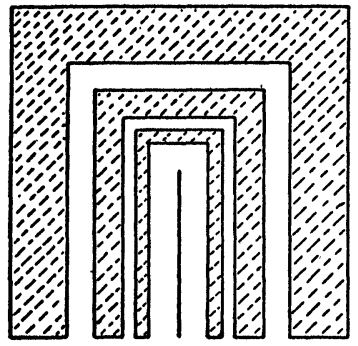

EXAMPLE 4-1

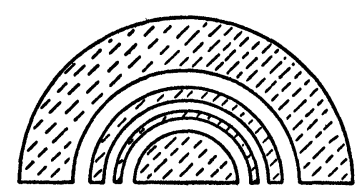

EXAMPLE 4-2

the $A_{i}$ converge. Consider Example 4. The shaded areas in Example 4.1 form a folded domain, while in Example 4.2 they do not-the reason being that the latter may be straightened out (by stretching) without doing violence to the topology.

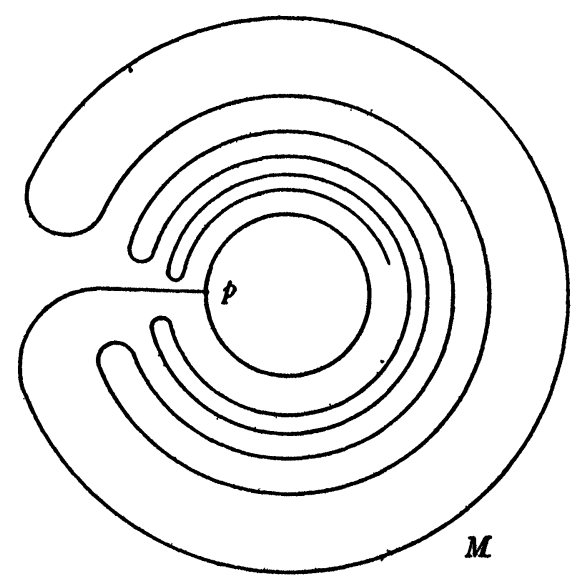

EXAMPLE 5

A cyclic $^{6}$ aposyndetic continuum in $S^{2}$ may be characterized as a continuum each of whose complementary domains is simple (i.e., has a simple closed curve for a boundary) such that this collection of simple domains contains no folded subcollection [5]. That is, the situation must be like Example 4.2, not Example 4.1. Wilder also has this theorem or something very analogous in his book [18].

In connection with the term cyclic, it is fortunate that aposyndetic continua have another characteristic in common with continuous curves:

- A continuum is said to be cyclic provided that no point of the continuum disconnects it. 
CutPoint Equivalence Theorem. In order that a point $p$ of an aposyndetic continuum $M$ be a separating point of $M$ it is necessary and sufficient that $p$ be a weak cutpoint of $M$ [14].

A point $p$ is a separating point (often called cutpoint) of a continuum $M$ provided that $M-p$ is not connected. A point $p$ is a weak cutpoint (often called cutpoint) of a continuum $M$ provided that $M-p$ is not strongly connected (i.e., continuum-wise connected). For continua in general the two properties are not the same. Consider Example 5. (In this example, if the circle were considered to be a point, $M$ would be a simple closed curve.) If $p$ is removed from $M$ the remaining set is connected but not strongly connected. For aposyndetic continua Whyburn (and I would guess Wilder also) showed that this cannot happen.

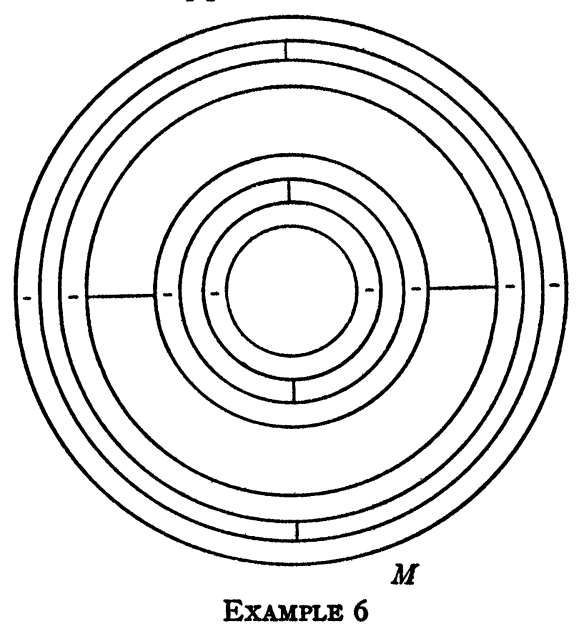

There are, however, considerable differences between continuous curves and aposyndetic continua. In Example 1, $M$ is aposyndetic at every point and connected im kleinen at $p$ but is not locally connected at $p$. In fact a cyclic continuum may be aposyndetic and not be connected im kleinen at any point whatsoever. In Example 6 the continuum $M$ consists of all circles centered on $(1 / 2,0)$ and passing through a point of the Cantor discontinuum (on the interval from 0 to 1 on the $x$-axis) together with the bridging arcs as indicated each of which is to be considered to be a point. Since these bridging arcs alternate their position between the vertical and horizontal (as the size of the gap bridged decreases) it is clear that $M$ is not connected im kleinen at any point of $M$. A little examination, however, shows that $M$ is aposyndetic at every point of $M$. 
Classification of continua. With the meaning of aposyndetic well in mind but before proceeding further we shall try to get a view of all continua in the light of this notion. If a person were to start to classify continua he would most certainly want the well-behaved continua (like arcs, simple closed curves, and continuous curves in general) at

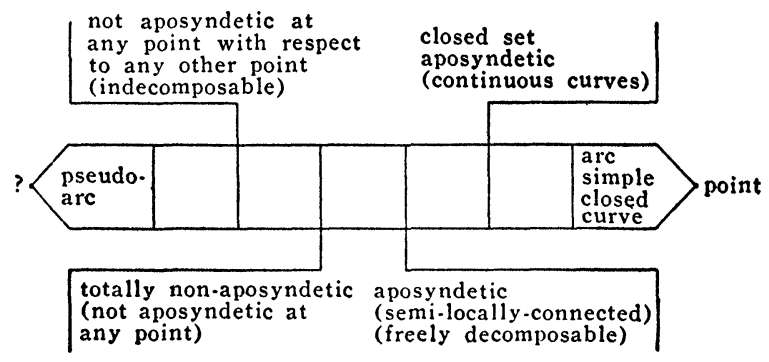

Spectrum

one end of the classification and the ill-behaved or psychopathic continua (like indecomposable continua) at the other. Imagine all continua spread out in a sort of spectrum with the bad ones on the left and the good ones on the right. This is illustrated in the figure. An indecomposable continuum is one which is not the sum of two proper subcontinua. One of the simplest examples of such a continuum is the one indicated in Example 7 [8]. This continuum consists of a countable infinity of collections of semi-circles, the first

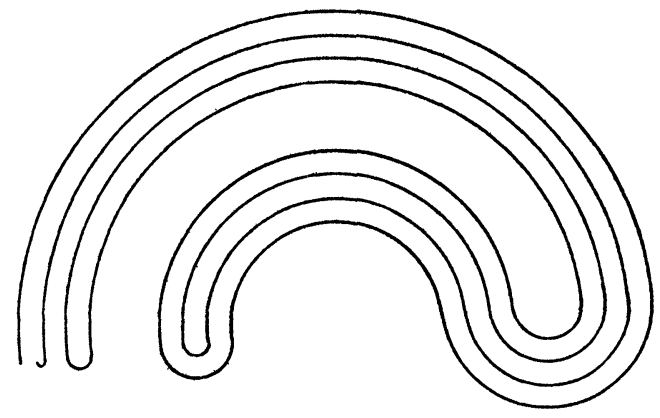

EXAMPLE 7

such collection being concentric at $(1 / 2,0)$ on the $X$-axis and using up all of the points of the Cantor discontinuum as endpoints of its elements, the second such collection being concentric at $(5 / 6,0)$ and using up all of the points of the right-hand half of the Cantor set as endpoints of its elements, etc., as indicated. Notice that in this 
example no point is a separating point but every point is a weak cutpoint.

Fundamental cutpoint theorems. In the left-hand half of the spectrum the weak cutpoint notion is of considerable importance. Actually we have two fundamental theorems.

THEOREM 1. A totally non-aposyndetic continuum contains a weak cutpoint [6]. (A totally non-aposyndetic continuum is one which is not aposyndetic at any one of its points.)

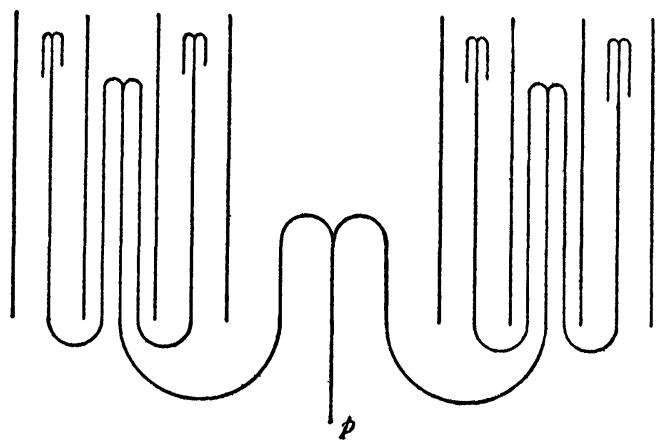

\section{EXAMPLE 8}

One is the largest number of weak cutpoints that a totally nonaposyndetic continuum must necessarily contain. For a simple case, consider again Example 5, and replace the ray (which starts at $p$ and is wrapped back and forth around the circle) by a bundle of rays (with a Cantor set cross-section) all of which start at p. A little examining will show that this continuum is totally non-aposyndetic. Nevertheless, it has no separating point and $p$ is its only weak cutpoint.

Theorem 2. A totally non-semi-locally-connected continuum possesses a dense set of weak cutpoints [6].

Theorem 2 might lead one to suspect that a totally non-semilocally-connected continuum is more non-aposyndetic than a totally non-aposyndetic continuum. This is not the case, for such a continuum need not be totally non-aposyndetic, in fact, such a continuum may be locally connected at one of its points. Consider Example 8. The continuum indicated in this example consists of vertical unit intervals erected at each point of the Cantor discontinuum on the interval from 0 to 1 of the $X$-axis (in the plane) together with the branching structure emanating from the point $p$. This 
branching structure consists of a bundle of rays (with a Cantor set cross-section) emanating from $p$ and separating into equal branches at the places indicated in the figure. The continuum thus obtained is totally non-semi-locally-connected, each of its points is a weak cutpoint, but it is locally connected at $p$.

Using these two theorems, one obtains a rather interesting theorem about continua which have no weak cutpoints.

TheOREM 3. A continuum which contains no weak cutpoint is both aposyndetic and s-l-c at all points of a dense inner limiting subset (G G $^{-s e t)}$.

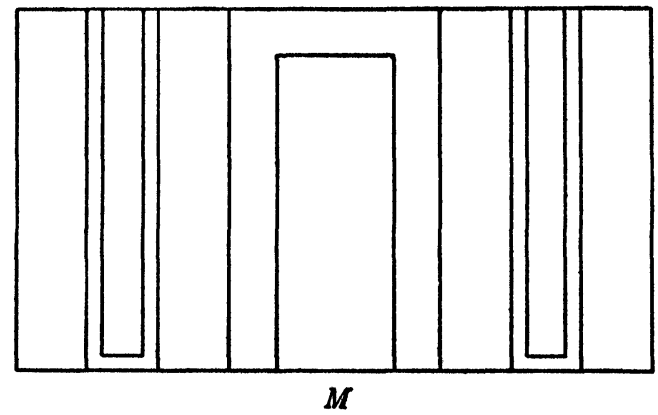

EXAMPLE 9

In spite of this theorem and Wilder's terminology (avoidability) a continuum containing no weak cutpoint may be composed largely of points at which it is neither aposyndetic nor s-l-c. To give a strikingly good example would take up too much space but the following example will suffice. In Example 9 the continuum $M$ consists of a rectangle (in the plane) together with the vertical intervals spanning the rectangle at the points of the Cantor discontinuum on its base plus infinitely many $U$-shaped arcs based alternately on the top and bottom sides of the rectangle as indicated. The continuum $M$ is cyclic but fails to be aposyndetic or semi-locally-connected at each nonendpoint of each of the vertical intervals.

I suspect these cutpoint theorems of having many applications. I want to mention one. Bing showed that if a nondegenerate continuum is cut (in the weak sense) by no one point but is cut by each pair of its points, then it is a simple closed curve. Using the first Fundamental Cutpoint Theorem he knew that the continuum had to be aposyndetic at a point and this gave him just enough of a hold on the problem to complete the proof [3].

Topological products. With the discussion and examples up to this 
point in mind, it would not be unreasonable to suspect that the topological product of two nondegenerate continua (it contains no weak cutpoint) should be aposyndetic. While this is not self-evident in the case of indecomposable continua, we do have the following:

Product Theorem (Whyburn-Jones). If $H$ and $K$ are nondegenerate continua their Cartesian product $H \times K$ is aposyndetic $[6 ; 14]$.

Whyburn first discovered this theorem but required one of the continua to be aposyndetic. There being so many products these days, it may be well to point out that the plane is the Cartesian product of the $X$-and $Y$-axis. An annulus is the product of an arc with a circle. So a totally non-aposyndetic continuum must be a kind of thin continuum: that is, it cannot be a topological product and it must contain a weak cutpoint.

Spectrum analysis. Looking at the spectrum of continua (see the figure) let us examine continua of various kinds and various properties of continua. Consider first arc-wise connectedness. Using the various examples that I have given, it is easy to see that arc-wise connected continua lie in every part of the spectrum except in the indecomposable end. So there is a problem of possibly expanding that portion of the spectrum where they must be arc-wise connected. Let us say that a continuum $M$ is $G$-aposyndetic if $G$ is a family of sets and for each point $p$ of $M$ and each element $g$ of $G$ lying in $M$ but not containing $p$, there exist a subcontinuum $H$ of $M$ and an open subset $U$ of $M$ such that $M-g \supset H \supset U \supset p$. Roughly, $M$ is bound together at each of its points away from each element of $G$. If $G$ is the collection of all closed sets, a continuum is $G$-aposyndetic if and only if it is locally connected and such continua are known to be arc-wise connected. If $G$ is the collection of degenerate sets, a continuum is $G$-aposyndetic if and only if it is aposyndetic and even in the plane such a continuum may be far from being arc-wise connected. Whyburn pointed this out with a different (and simpler) example [14] but the reader may see this by looking at Example 6 .

Now consider continua in a given 2-sphere, $S^{2}$, and suppose that we let $G_{1}$ be the family of all continua in $S^{2}$. Let $G_{2}$ be the family of all continua in $S^{2}$ that are $G_{1}$-aposyndetic. In general, for each positive integer, $i$, let $G_{i+1}$ be the family of all continua in $S^{2}$ which are $G_{i}$-aposyndetic. For each $i>1$, each element of $G_{i}$ is aposyndetic. Those continua in $G_{1}$ do not have to be arc-wise connected. Those in $G_{2}$ must all be arc-wise connected. When I first discovered this, I thought that perhaps here was an extension of the arc-wise connected- 
ness theorem. But no-the elements of $G_{2}$ are continuous curves! What is the nature of the elements of $G_{3}$ ? While they are all aposyndetic, they are not all arc-wise connected. Consider Example 10.

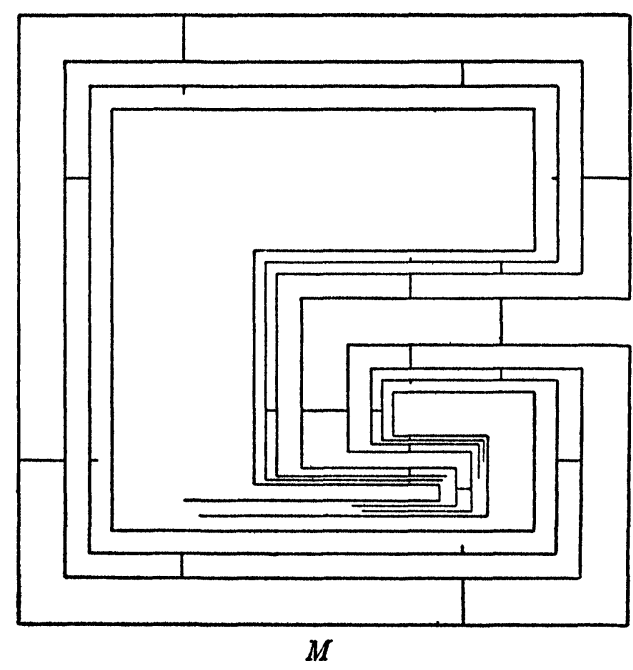

EXAMPLE 10

Start with a square (in the plane) plus its interior. Using the method of Yoneyama [19] construct an indecomposable continuum by digging a canal (i.e., removing a connected open set) into this square that winds back and forth within the square so as to come arbitrarily close to every point of what is left by a sufficiently devious path. Then add to this continuum infinitely many open curves which have been drawn into the entire length of the canal and which have been bridged with arcs as indicated, the distance between such bridges being roughly equal to the width of the canal at the point of the bridge. Call this continuum $M$. To get from the open curve part of $M$ to the indecomposable part of $M$ with an arc (or continuous curve) one either has to oscillate too much because of the staggered nature of the bridges or go too far down the canal (its entire length in fact) in trying to reduce the distance between bridges. Therefore the elements of $G_{2}$ (continuous curves) lying in the continuum $M$ must lie wholly within its indecomposable (Yoneyama) subcontinuum or entirely outside of this subcontinuum. With this in mind one can see that $M$ is $G_{2}$-aposyndetic but not arc-wise connected. A simpler example than Example 10 will not suffice because from the WilderWhyburn Boundary Theorem those elements of $G_{2}$ possible in a given 
$G_{3}$-aposyndetic continuum will consist, in addition to degenerate and possibly other sets, of the boundaries of its complementary domains. For instance in Example 6, $M$ (which is not arc-wise connected) is not $G_{2}$-aposyndetic.

Now, how about the elements of $G_{4}$ ? Are they all arc-wise connected? By elementary (and facetious) induction, when $i$ is odd the elements of $G_{i}$ do not have to be arc-wise connected, and when $i$ is even the elements of $G_{i}$ are arc-wise connected. So the only problem here is in the limit: Does $\operatorname{Lim}_{n \rightarrow \infty} G_{2 n+1}=\operatorname{Lim}_{n \rightarrow \infty} G_{2 n}$ ? And are the elements of the limit collection arc-wise connected? As a matter of fact my knowledge does not extend to $G_{4}$ but perhaps this view of the problem will tempt someone else to investigate $G_{i}, i>3$.

Thus for arc-wise connectedness, looking at the spectrum and attempting to further refine its structure has not yet proved fruitful (except to learn that aposyndetic continua in $S^{2}$ do have to contain arcs and that elements of $G_{2}$ are continuous curves).

Now consider irreducible continua. Whyburn showed that those which are aposyndetic must be arcs [14]. If they are non-aposyndetic, they may be scattered anywhere in that portion of the spectrum.

Consider a continuum $M$ which is topologically equivalent to each of its nondegenerate subcontinua. If $M$ is aposyndetic, it is an arc because it is irreducible between some pair of its points. If $M$ is non-aposyndetic it may lie at the other end of the spectrum (as shown by Moise's example of a pseudo-arc) [9]. But must it? This question, perhaps not quite in this form, has been unanswered for a long time, and I suspect that a good many more division lines will have to be added to the spectrum before an answer can be obtained.

Consider a homogeneous continuum $M$ in $S^{2}$. If $M$ is aposyndetic, it must be a simple closed curve [7]. If $M$ is not aposyndetic (and such continua do exist $[2 ; 10])$, two cases arise: (1) If $M$ does not separate $S^{2}, M$ is indecomposable; $;^{7}(2)$ if $M$ does separate $S^{2}$, I would guess it may exist outside of the indecomposable end of the spectrum but I am not quite satisfied about this.

The number of problems that this scheme of spectrum analysis calls to mind, even for continua in the plane, is immense. I shall mention two more:

1. What does it take to push a totally non-aposyndetic continuum into the indecomposable end of the spectrum?

2. Is there a continuum at the extreme left end of the spectrum which if used as a "point" would decompose $S^{2}$ into itself? From an

\footnotetext{
7 The proof of this will appear elsewhere.
} 
esthetic point of view, the answer to this question should be yes, in order that the spectrum return to its starting point. ${ }^{8}$

I have not touched upon dimension theory at all. I would like to point out that one cannot raise the dimension of a Knaster continuum (and keep it a Knaster continuum) by means of Cartesian products for then they become aposyndetic. ${ }^{9}$

As I indicated in the introduction, we have here only a beginning of a classification of continua by means of their aposyndetic properties. I have indicated one way, but not a very fruitful way, of refining this classification. And I have indicated in a rough sort of way how this crude classification may be used not only to suggest new problems about continua but also to view the class of continua as a whole. I hope that this discussion will challenge others to work on these problems, but the research worker who expects to lead a life of ease should stay out of the left end of the spectrum.

\section{BIBLIOGRAPHY}

1. W. L. Ayres, On avoidable points of continua with an application to end points, Math. Zeit. vol. 34 (1931) pp. 161-178.

2. R. H. Bing, A homogeneous indecomposable plane continuum, Duke Math. J. vol. 15 (1948) pp. 729-742.

3. - Some characterizations of arcs and simple closed curves, Amer. J. Math. vol. 70 (1948) pp. 497-506.

4. F. B. Jones, Aposyndetic continua and certain boundary problems, Amer. J. Math. vol. 63 (1941) pp. 545-553.

5. - A characterization of a semi-locally-connected plane continuum, Bull. Amer. Math. Soc. vol. 53 (1947) pp. 170-175.

6. - Concerning non-aposyndetic continua, Amer. J. Math. vol. 70 (1948) pp. $403-413$.

7. - A note on homogeneous plane continua, Bull. Amer. Math. Soc. vol. 55 (1949) pp. 113-114.

8. C. Kurotowski, Théorie des continus irreductible entre deux points I, Fund. Math. vol. 3 (1922) pp. 200-231.

9. E. E. Moise, An indecomposable plane continuum which is homeomorphic to each of its non-degenerate subcontinua, Trans. Amer. Math. Soc. vol. 63 (1948) pp. 581-594.

10. — A note on the pseudo-arc, Trans. Amer. Math. Soc. vol. 67 (1949) pp. 57-58.

11. R. L. Moore, Foundations of point-set theory, Amer. Math. Soc. Colloquium Publications, vol. 13, New York, 1932.

12. A. Schoenflies, Die Entwickelung der Lehre von den Punktmannigfaltigkeiten, Leipzig, 1908, p. 237.

8 This question has been answered by R. D. Anderson in the affirmative.

${ }^{\theta}$ Bing has raised the dimension of a Knaster continuum but by another method. His paper Higher-dimensional hereditarily indecomposable continua appears in Trans. Amer. Math. Soc. vol. 71 (1951) pp. 267-273. 
13. Marie Torhorst, Über den Rand der einfach zusammenhängenden ebenen Gebiete, Math. Zeit. vol. 9 (1921) pp. 44-65.

14. G. T. Whyburn, Semi-locally-connected sets, Amer. J. Math. vol. 61 (1939) pp. 733-749.

15. - Analytic topology, Amer. Math. Soc. Colloquium Publications, vol. 28, New York, 1942.

16. R. L. Wilder, Sets which satisfy certain avoidability conditions, Casopis pro Pestovani Mathematiky a Fysiky vol. 67 (1938) pp. 185-198.

17. - Property $S_{n}$, Amer. J. Math. vol. 61 (1939) pp. 823-832.

18. - Topology of manifolds, Amer. Math. Soc. Colloquium Publications, vol. 32, New York, 1949.

19. K. Yoneyama, Theory of continuous sets of points I, Tôhoku Math. J. vol. 12 (1917) pp. 43-153.

The University of Texas AND

The University of North Carolina 